\title{
MATHEMATICAL PERFORMANCE ANALYSIS OF A TEMPERATURE CONTROLLED BULK STORAGE ROOM
}

\author{
Simon van Mourik* Johan P.M. Ploegaert** \\ Hans Zwart ${ }^{* * *}$ Karel J. Keesman ${ }^{* * * *}$ \\ * Department of Applied Mathematics, University of \\ Twente, The Netherlands, Phone: +31 (53) 4893473 , \\ Fax:+31(53)4893800,e-mail: s.vanmourik@utwente.nl \\ ** Systems \& Control Group, Wageningen University, The \\ Netherlands \\ *** Department of Applied Mathematics, University of \\ Twente, The Netherlands \\ **** Systems \& Control Group, Wageningen University, \\ The Netherlands
}

\begin{abstract}
Usually, control design takes place after the plant has been designed. However, the performance of the plant connected to the controller might be improved by simultaneous design of the plant and the controller. In this paper, expressions are deduced that describe the dynamics of a controlled plant (a bulk storage room) and its design criteria. These expressions contain all the physical system parameters, and give insight on how the system performance (in terms of energy usage and temperature uniformity) depends on the design parameters (these are the bulk height and the ventilator- and heat exchanger capacity). This insight gives guidelines for optimal storage room design. The physical relations that could not be found in the literature were identified experimentally. Copyright (c)2007 IFAC
\end{abstract}

Keywords: Control, process optimization, modelling, identification.

\section{INTRODUCTION}

A large volume of perishable foods that are stored consists of bulk stored foods. The temperature of the products is usually kept at a low value to maintain product quality. In general, the temperature control is done in two ways; ventilation with outside air, or by means of a heat exchanger. Furthermore, a ventilator enforces the air circulation. Cold air flows usually upwards through the bulk. Inside the bulk, the air warms up and consequently the products at the top will be some degrees warmer than those at the bottom, see Rastovski and van Es et al [1987]. A larger airflow will decrease these spatial variations, but will be costly. Model-based control design is a nontrivial task, since a standard model that describes the time- and spatially dependent temperatures will consist of a set of nonlinear partial differential equations. Systems design is strongly correlated to controller design. The controller adds dynamics to the system, causing it to behave differently than the uncontrolled system. For storage room design, it is therefore desirable to design the plant and controller simultaneously, instead of separately. There is extensive literature available on the modelling aspects of bulk storage rooms, for example $\mathrm{Xu}$ and Burfoot [1999b,a]; Lukasse et al. [2006]; 
Verdijck [2003]. The main goal in these papers is to gain insight in the dynamics of temperature and humidity. The literature on model-based control design for bulk stored food products is limited. In Keesman et al. [2003] and Verdijck [2003], model predictive control (MPC) algorithms were used for the temperature and humidity control of a bulk storage room with outside air ventilation. Both proposed algorithms are model based and were tested by simulation studies. The aim of the algorithms is to drive temperature and humidity to a desired set point at low economic costs. Due to the relatively high complexity of the models, model-based control design requires computer simulations that are very time consuming. This is due to the fact that the controller is constructed numerically for one particular system configuration, so controller design has to be carried out for each different system configuration. Optimal design of a climate controlled bulk storage room would be very time consuming, and is not shown in the works discussed above. In fact, the authors could not find literature on optimal design of a controlled storage room where not only control parameters are adjusted, but also physical system parameters.

In this paper, a different approach is used, starting with the results in Van Mourik et al. [2006], where a basic physical model was derived and validated with experimental results. An open loop control law, which explicitly depends on all the physical model parameters, was successfully constructed. The controller determines the times when to switch between two discrete inputs. This input switching is realistic since often in practice the ventilator is switched on and off on a regular basis. The reason for the choice of the simple structure of an open loop controller is the following. The controller as well as the performance criteria that are presented in section 3 , are closed expressions that contain all the prior physical knowledge, and are therefore easy to analyze. For the sake of simplicity, no disturbances were modelled. Since some analytical relations could not be found in the literature, they are identified experimentally in section 4. These relations describe the energy usage of the ventilator, and the effectiveness of the heat exchanger, both as functions of the airflow. The relationship between each design criterium and the design parameters consists of a single expression and is therefore easily computed in section 5 .

\section{THE MODEL}

\subsection{Bulk storage room model}

The storage room model is divided into two parts, namely the shaft and the bulk, see Figure 1.
We consider the (approximated) model that was

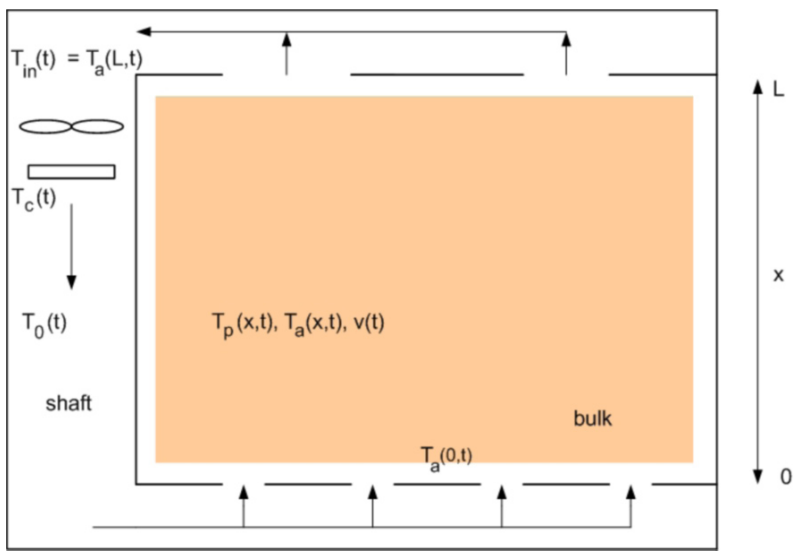

Figure 1. Schematic representation of a bulk storage room.

proposed in Van Mourik et al. [2006].

$$
\begin{aligned}
& V \frac{\partial T_{0}(t)}{\partial t}=-\Phi \alpha(\Phi)\left(T_{a}(L, t)-T_{c}(t)\right)+ \\
& \Phi T_{a}(L, t)-\Phi T_{0}(t) \\
& \frac{\partial T_{a}(x, t)}{\partial t}=-v \frac{\partial T_{a}(x, t)}{\partial x}+ \\
& M_{4}\left(T_{p}(x, t)-T_{a}(x, t)\right) \\
& \frac{\partial T_{p}(R, x, t)}{\partial t}=A_{p} T_{p}(R, x, t)+B_{p} T_{a}(x, t) \\
& T_{a}(0, t)=T_{0}(t) .
\end{aligned}
$$

Equations (1)-(4) are referred to as the nominal model. Equation (1) describes the temperature dynamics inside the shaft. $V$ is the volume of the shaft, $T_{a}(L, t)$ the air temperature at the top of the bulk, $T_{c}(t)$ the temperature of the cooling element inside the heat exchanger, $\rho_{a}$ the air density, $c_{a}$ the heat capacity of air, and $\Phi$ the air flux. The dimensionless function $\alpha(\Phi)$ denotes the effectiveness of the cooling device: $\alpha(\Phi)=1$ implies that the incoming air $T_{i n}(t)$ is totally cooled down (or heated up) to $T_{c}(t)$, while $\alpha(\Phi)=0$ implies that the incoming air is not cooled at all. The relation between $\Phi$ and $\alpha(\Phi)$ will be determined experimentally later on. Equation (2) describes the temperature dynamics of the air inside the bulk. The two r.h.s. terms in equation (2) denote the convection of heat and the heat exchange between product surface and air, respectively. Here, $x$ denotes the height in the bulk, that varies from 0 to $L$. Further, $M_{4}=\frac{h(v) A_{p}}{\gamma \rho_{a} c_{a}}$, with $\gamma$ the bulk porosity, and $A_{p}$ the product surface area per bulk volume. The heat transfer coefficient $h(v)$ depends on $v$ via the implicit relation (see Xu and Burfoot [1999b])

$$
\mathrm{Nu}=\left(0.5 \operatorname{Re}^{1 / 2}+0.2 \operatorname{Re}^{2 / 3}\right) \operatorname{Pr}^{1 / 3}
$$

for $10<\operatorname{Re}<10^{4}$, with $\mathrm{Nu}, \operatorname{Re}$ and $\operatorname{Pr}$ the Nusselt, Reynolds and Prandtl number, see the Appendix. The average velocity inside the bulk is $v=\frac{\Phi}{A_{f} \gamma}$, with $A_{f}$ the area of the bulk floor. 
Equation (3) describes the temperature dynamics of the product skin (which represents the product temperature) at height $x$ inside the bulk. Here, $A_{p}=-a_{2} / a_{3}, B_{p}=a_{1} / a_{3}$, and $a_{1}, a_{2}$ and $a_{3}$ are listed in the Appendix. Parameters $a$ and $\mathrm{Bi}$ are the heat production of the products and the Biot number. The most important assumptions are:

- The air- and product temperature in the bulk, $T_{a}$ and $T_{p}$, only vary with the height of the bulk, and so not with the width;

- There is no heat transport through the walls;

- The air temperature in the shaft, $T_{0}(t)$, is well-mixed and therefore uniform over the length of the shaft;

- No moisture transport is incorporated;

- Heat diffusion in the air is neglected.

In Van Mourik et al. [2006] the model predictions were found to be accurate when compared to experimental results.

\subsection{Open loop control}

The algorithm for an open loop control law in Van Mourik et al. [2006] is followed. Three simplifying steps were taken.

(1) The inputs $T_{c}(t)$ and $\Phi(t)$ are assumed to switch between the values $\left(T_{c, 1}, T_{c, 2}\right)$ and $\left(\Phi_{1}, \Phi_{2}\right)$ respectively. Between two switching times our model (1)-(4) is linear. The switching interval is in the order of ten minutes.

(2) The air temperatures $T_{0}(t)$ and $T_{a}(x, t)$ have very fast dynamics, and hence they settle immediately after an input switch.

(3) The dynamics of the product temperature is slow, and hence can be well approximated by a first order system.

From the simplified model an analytic expression for the open loop control law was derived. On every time interval $\left(0, \tau_{f}\right)$, the input pair is switched at $t=\tau_{\text {opt }}$, with

$$
\tau_{o p t}=\frac{-\tau_{f}\left(A_{p, 2}^{*} T_{p, o p t}+B_{p, 2}^{*} T_{c, 2}\right)}{\left(A_{p, 1}^{*}-A_{p, 2}^{*}\right) T_{p, o p t}+B_{p, 1}^{*} T_{c, 1}-B_{p, 2}^{*} T_{c, 2}},
$$

with

$$
\begin{aligned}
A_{p}^{*} & =\frac{\tilde{A}_{p} A_{p}}{A_{p}+\tilde{A}_{p}} \\
B_{p}^{*} & =-\frac{\tilde{B}_{p} B_{p}}{A_{p}+\tilde{A}_{p}} \\
\tilde{A}_{p} & =-\frac{A_{p}^{2}}{M_{5} B_{p}}+\frac{A_{p}^{2}(1-\alpha)}{M_{5} B_{p}} \exp \left(M_{5}\left(\frac{B_{p}+A_{p}}{-A_{p}}\right)\right) \\
\tilde{B}_{p} & =\frac{\alpha A_{p}^{2}}{M_{5} B_{p}} \exp \left(M_{5}\left(\frac{B_{p}+A_{p}}{-A_{p}}\right)\right) .
\end{aligned}
$$

The subscripts 1 and 2 in (5) denote the discrete input. $M_{5}$ is listed in the Appendix. $T_{p, o p t}$ is the optimal product temperature. The switching time $\tau_{\text {opt }}$ contains all prior physical knowledge of the system. Simulation showed that this control algorithm accurately drives $T_{p}(L, t)$ in the nominal model to its desired value (see Figure 2 ).

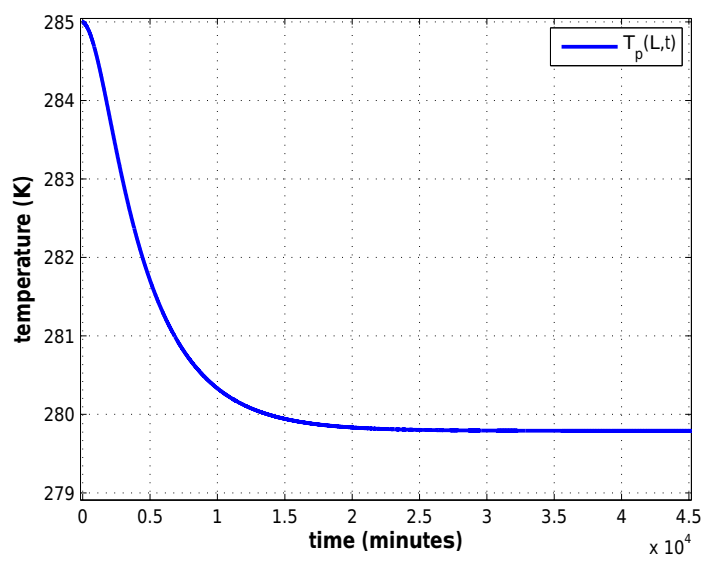

Figure 2. From an arbitrary initial value of $285 K$, the product temperature at the top of the bulk converges to its optimal value of $280 \mathrm{~K}$. The accuracy is $0.2 \mathrm{~K}$.

\section{PERFORMANCE CRITERIA}

The objective in this section is to derive criteria which indicate the performance of the controlled system. These criteria are the energy use of the fan and of the heat exchanger, and the uniformity of the bulk temperature.

\subsection{Energy costs of the ventilator and heat exchanger}

The average energy demand of the ventilator (in $W)$ is

$$
E_{\text {vent }}=\frac{\tau E_{\text {vent }, 1}+\left(\tau_{f}-\tau\right) E_{\text {vent }, 2}}{\tau_{f}} .
$$

Here, $E_{\text {vent }, 1}$ and $E_{\text {vent }, 2}$ denote the energy demands for the discrete values of $\Phi$, and $\tau$ is the switching time. The relation between $E_{\text {vent }}$ and $\Phi$ is determined experimentally in section 4 . The average energy demand of the heat exchanger was not measurable, and since a straightforward model was not found in the literature, the heat exchanger is assumed to have a constant yield of $y=50 \%$. The average energy usage in the time interval $\left(0, \tau_{f}\right)$ is

$$
\begin{aligned}
E_{h e} & =\frac{1}{y \tau_{f}}\left(\tau \rho_{a} c_{a} \Phi_{1} \alpha_{1}\left(T_{a}(L)-T_{c, 1}\right)\right. \\
& \left.+\left(\tau_{f}-\tau\right) \rho_{a} c_{a} \Phi_{2} \alpha_{2}\left(T_{a}(L)-T_{c, 2}\right)\right),
\end{aligned}
$$


where the subscripts denote the relations with the input values. We assumed that $T_{a}(L, t)$ is constant during this time interval.

\subsection{Temperature differences inside the bulk}

A large temperature difference between the products at the top and at the bottom of the bulk is undesirable. We are particularly interested in the difference

$$
\Delta T_{p}=T_{p}(L, t)-T_{p}(0, t),
$$

after the controller has driven the bulk temperature to a steady profile. Because the controller will drive $T_{p}(L, t)$ to $T_{p, o p t}$, we only need an expression for $T_{p}(0, t)$ to determine equation (8). The derivation is omitted, but is done in a similar way as in Van Mourik et al. [2006], and resulted in

$$
T_{p}(0, t)=-\frac{\tau B_{p 0,1} T_{c, 1}+\left(\tau_{f}-\tau\right) B_{p 0,2} T_{c, 2}}{\tau A_{p 0,1}+\left(\tau_{f}-\tau\right) A_{p 0,2}},
$$

where the subscripts 1 and 2 denote the relations with the input values. We have that $A_{p 0}=\frac{A_{0}^{*} A_{p}}{A_{0}^{*}+A_{p}}$ and $B_{p 0}=\frac{-B_{0}^{*} B_{p}}{B_{0}^{*}+B_{p}}$, with $A_{0}^{*}=\tilde{A}_{p} / M_{6}$ and $B_{0}^{*}=$ $\tilde{B}_{p} / M_{6}$, and $M_{6}$ is listed in the Appendix. A simulation study showed that this prediction is accurate.

\section{MODEL IDENTIFICATION BY EXPERIMENT}

The model and performance criteria are still incomplete at three points. No model nor product specifications were found that relates pressure differences over a ventilator with its variable energy usage in a straightforward way. Further, the pressure drop over the ventilator equals the sum of the pressure drops over the different flow channels, such as the ventilator shaft and the bulk. Although the Bernoulli equations relates the pressure drop to the airflow rate, they are practically unfeasible for irregular obstacles in a standard storage room, like for example T-bar floors. Finally, a model for $\alpha(\Phi)$ has not been found in the literature. The above relations are therefore determined by experimental identification. The experimental setup consisted of a closed storage container with inner dimensions $3.6 \times$ $2.9 \times 2.1 \mathrm{~m}$. The ventilator in the experimental storage room is placed after the heat exchanger, instead of before like in Figure 1, but this does not alter the nominal model. The ventilator was of type ITHO K315LTW, and the heat exchanger was of type Helpman LEX 22E. The data were measured with Campbell CR10 data loggers, and the airflows were measured with a Lambrecht 642 anemometer.

\subsection{Identification of ventilator energy demands}

There was no bulk of foodstuffs placed in the storage room. The relation between the pressure drop $\Delta P$ over a storage room with a bulk, and the air flux is

$$
\Delta P=\Delta P_{b u l k}+\Delta P_{\text {empty }},
$$

with $\Delta P_{\text {bulk }}$ and $\Delta P_{\text {empty }}$ the pressure drops over the bulk and over the empty storage room (without a bulk), respectively. The relation between pressure difference and ventilator energy demands was determined by measuring the average pressure over the ventilator and its energy usage, for 5 ventilator stages, each one creating a different air flux. For each stage, the online measurements were carried out with an interval time of one minute, for half an hour. Using a least squares method, the best fit between ventilator energy demand and pressure drop over the ventilator was

$E_{\text {vent }}=61.80+23.36 \Delta P-1.05 \Delta P^{2}+0.02 \Delta P^{3}$.

The fit has a low standard deviation for $\Delta P \in$ $[5 P a, 25 P a]$, which is the pressure range of the ventilator. The pressure drop in the empty storage room was fitted to the air flow rate as

$$
\Delta P_{\text {empty }}=-11.66+94.86 \Phi,
$$

and shows a low standard deviation for $\Phi \in$ $\left[0.1 \mathrm{~m}^{3} / \mathrm{s}, 0.34 \mathrm{~m}^{3} / \mathrm{s}\right]$, the flux range of the ventilator. For a bulk of potatoes that is placed in the storage room, the pressure drop over a bulk of spherical products is described by the Ergun formula (see Ergun [1952])

$\Delta P_{b u l k}=L\left(150 \frac{(1-\gamma)^{2} \mu}{\gamma^{3}(2 R)^{2}} v+1.75 \frac{(1-\gamma) \rho_{a}}{\gamma^{3} 2 R} v^{2}\right)$.

Here, $R$ is the product radius, and $\mu$ the dynamic viscosity of the air.

\subsection{Relation between $\alpha$ and $\Phi$}

For six different stages of the ventilator, the air temperatures right before and right after the heat exchanger were measured every minute for at least half an hour. Also the air fluxes and the temperature of the cooling element inside the heat exchanger were measured online. In equilibrium the air temperatures satisfy

$$
0=(1-\alpha(\Phi)) T_{a}(L, t)-T_{0}(t)+\alpha(\Phi) T_{c}(t) .
$$

$\alpha(\Phi)$ is derived from this equation by inserting the averages of the measured temperatures over time for each ventilator stage, and taking a least squares fit. $T_{c}$ is taken as the mean of the temperatures at the locations of the incoming and outgoing cooling liquid. A least squares fit resulted in

$$
\alpha(\Phi)=-0.95 \Phi+1.04
$$

for $\Phi \in\left[0.1 \mathrm{~m}^{3} / \mathrm{s}, 0.34 \mathrm{~m}^{3} / \mathrm{s}\right]$ 


\section{PERFORMANCE ANALYSIS}

To visualize the relation between the performance and the design parameters, we consider here a simplified situation. We assume that $\tau=\tau_{o p t}$, at all time intervals. This implies that the temperature dynamics are equal within each time interval $\left(0, \tau_{f}\right)$. This means that the controlled system is undisturbed and in steady state. Further, we neglected the small deviations of $T_{a}(L, t)$ in equation (7). The steady state value corresponding to $\tau=\tau_{\text {opt }}$ is

$$
T_{a}(L, t)=-\frac{\tau \tilde{B}_{1} T_{c, 1}+\left(\tau_{f}-\tau\right) \tilde{B}_{2} T_{c, 2}}{\tau \tilde{A}_{1}+\left(\tau_{f}-\tau\right) \tilde{A}_{2}},
$$

where the subscripts 1 and 2 denote the relations with the input values. A simulation study showed that this prediction is accurate. Equations (6)(8) now define the system performance, and they depend on equations (5) and (9)-(15). The system performance is now approximated by variation of design parameters from their nominal values. The nominal parameter values are marked with an asterisk (see Table 1), and they represent a storage room with a bulk of potatoes. For this

\begin{tabular}{|cc|cc|}
\hline$\Phi_{1}^{*}$ & $0.2 \mathrm{~m}^{3} / \mathrm{s}$ & $\Phi_{2}^{*}$ & $0.002 \mathrm{~m}^{3} / \mathrm{s}$ \\
$L^{*}$ & $2 \mathrm{~m}$ & $A_{f} \times L$ & $40 \mathrm{~m}^{3}$ \\
$R^{*}$ & $2.510^{-2} \mathrm{~m}$ & $V^{*}$ & $10 \mathrm{~m}^{3}$ \\
$\lambda_{p}^{*}$ & $0.55 \mathrm{~W} / \mathrm{mK}$ & $\rho_{p}^{*}$ & $1014 \mathrm{~kg} / \mathrm{m}^{3}$ \\
$a^{*}$ & $3.110^{-5} \mathrm{~W} / \mathrm{kg} \mathrm{K}$ & $A_{p}^{*}$ & $40 \mathrm{~m}^{2}$ \\
$\gamma^{*}$ & $0.3 \mathrm{~m}^{3} / \mathrm{m}^{3}$ & $c_{p}^{*}$ & $3.610^{3} \mathrm{~J} / \mathrm{kg} \mathrm{K}$ \\
$T_{c}^{*}$ & $275 \mathrm{~K}$ & $c_{a}^{*}$ & $210^{3} \mathrm{~J} / \mathrm{kg} \mathrm{K}$ \\
\hline
\end{tabular}

Table 1. Nominal parameter values for a bulk with potatoes.

analysis, the design parameters are chosen as $L, \Phi$, and $T_{c}$. Notice that these parameters also appear in the control law (5). We choose $\Phi_{2}=0.002$, and $E_{\text {vent }, 2}=0$, which corresponds to natural convection and this does not costs any ventilator energy. Further, $T_{c}$ is assumed to be constant throughout, so $T_{c, 1}=T_{c, 2}$, that is, no switching of the temperature of the cooling device. The bulk volume, $A_{f} \times L$, is always constant, so a higher bulk implies a smaller bulk floor area. We have the extra conditions that $\Phi$ and $\Delta P$ lie the experimental ranges $0.1 \mathrm{~m}^{3} / \mathrm{s} \leq \Phi \leq$ $0.35 \mathrm{~m}^{3} / \mathrm{s}$, and $5 P a \leq \Delta P \leq 25 P a$. To satisfy them, we constricted $L \in[1.8 \mathrm{~m}, 3.7 \mathrm{~m}], T_{c} \in$ $[260 \mathrm{~K}, 278 \mathrm{~K}]$, and $\Phi \in\left[0.16 \mathrm{~m}^{3} / \mathrm{s}, 0.34 \mathrm{~m}^{3} / \mathrm{s}\right]$.

\subsection{Energy usage}

The total energy used by the ventilator and the heat exchanger is $E$. The relations between $E$ and the design parameters, are numerically evaluated in the nominal parameter values, and turned out to be approximately equal to (see Figure 3)

$$
\begin{aligned}
E & =1.07\left(L-L^{*}\right)+6.40\left(T_{c}-T_{c}^{*}\right) \\
& +0.32\left(T_{c}-T_{c}^{*}\right)^{2}+E^{*},
\end{aligned}
$$

with $E^{*}=250.5 \mathrm{~W}$. The energy usage depends on $\Phi_{1}$ very weakly, and differed less than $1 W$ for $\Phi \in\left[0.16 \mathrm{~m}^{3} / \mathrm{s}, 0.34 \mathrm{~m}^{3} / \mathrm{s}\right]$. This may sound surprising. Indeed, for higher $\Phi_{1}$ the pressure drop rises, and so does the ventilator energy usage. But on the other hand, the ventilator will blow shorter for each time interval. A local minimum in $E$ is attained for $T_{c}=265 \mathrm{~K}$.

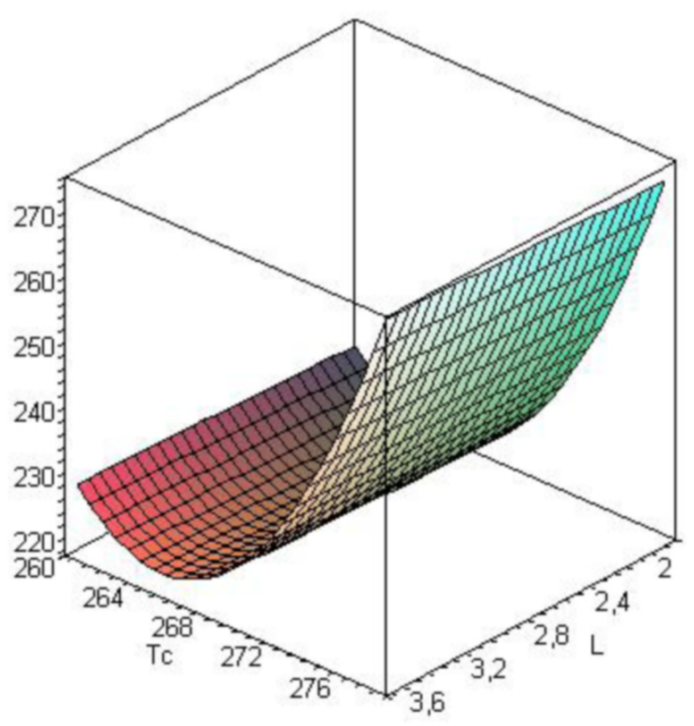

Figure 3. The energy usage is strongly correlated to $T_{c}$, and attains a local minimum for $T_{c}=$ $265 K$

\subsection{Temperature uniformity}

The temperature difference inside the bulk has the following correlation

$$
\begin{aligned}
\Delta T_{p} & =-0.56\left(\Phi_{1}-\Phi_{1}^{*}\right) \\
& -0.98\left(T_{c}-T_{c}^{*}\right)+4.49 .
\end{aligned}
$$

The temperature difference depends very weakly on $L$, less than $0.1 K$ for $L \in[1.8 \mathrm{~m}, 3.7 \mathrm{~m}]$. We see that an increase in ventilator capacity will result in a smaller temperature difference, and that a cooler cooling device will give a greater temperature difference.

\section{CONCLUSIONS}

For a temperature controlled bulk storage room an open loop control law and system performance indices were derived as explicit functions of the physical system properties. Hence, there is a clear connection between plant and controller design. To visualize things, the system performance was 
numerically determined as a function of the design parameters, under some simplifying assumptions. For the system with our choice of nominal parameters, the performance analysis showed the following tradeoff. The total energy usage is minimized by a low temperature of the cooling device, but the temperature difference over the bulk is minimized by a high temperature of the cooling device. Further, the temperature difference is decreased by a more powerful ventilator. However, the tradeoff here is that such a ventilator will be more expensive in purchase. The energy usage is decreased by a lower bulk. The tradeoff is that for a fixed bulk volume, a lower bulk means a larger floor area, which is usually more expensive than a higher roof.

The modelling of the system and the design criteria was done analytically. However, since not all expressions were found in literature, the missing relations were experimentally identified. For optimization purposes one should keep in mind that the identified relations are only valid on a limited parameter range.

Extension of the model, for example with heat loss through walls, will be straightforward but laborious. This is also true for dynamical disturbances and perturbations, where probably simulation studies of the nominal model and a feedback controller will be needed.

\subsection{Acknowledgement}

This work was supported by the Technology foundation STW under project number WWI.6345.

\section{REFERENCES}

S. Ergun. Fluid flow through packed columns. Chemical Engineering Progress, 48:89-94, 1952.

K.J. Keesman, D. Peters, and L.J.S. Lukasse. Control Engineering Practice, 11:505-516, 2003.

L.J.S. Lukasse, J.E. de Kramer-Cuppen, and A.J. van der Voort. A physical model to predict climate dynamics in ventilated bulk-storage of agricultural produce. accepted for International Journal of Refrigeration, 2006.

A. Rastovski and A. van Es et al. Storage of potatoes, Post-harvest behaviour, store design, storage practice, handling. Pudoc Wageningen, 1987.

S. Van Mourik, H.J. Zwart, and Keesman K.J. Analytic control law for a food storage room. Submitted, 2006, 2006.

G.J.C. Verdijck. Product quality control. PhD thesis, University of Eindhoven, 2003.

Y. Xu and D. Burfoot. Predicting condensation in bulks of foodstuffs. Journal of food engineering, 40:121-127, 1999a.
Y. Xu and D. Burfoot. Simulating the bulk storage of foodstuffs. Journal of food engineering, 39:23-29, 1999b.

\section{APPENDIX}

$\Phi \quad$ air flow through shaft $\left(\mathrm{m}^{3} / \mathrm{s}\right)$

$\alpha \quad$ cooling effectiveness $(K)$

$\alpha_{t h} \quad$ thermal diffusivity of air $\left(1.8710^{-5} \mathrm{~m}^{2} / \mathrm{s}\right)$

$\gamma \quad$ porosity $\left(m^{3}\right.$ air $/ m^{3}$ bulk $)$

$\lambda_{a} \quad$ conduction of air $\left(2.4310^{-2} \mathrm{~W} / \mathrm{m} \mathrm{K}\right)$

$\lambda_{p} \quad$ conduction of product $(W / m K)$

$\nu \quad$ kinematic viscosity of air $\left(1.346510^{-5} \mathrm{~m}^{2} / \mathrm{s}\right)$

$\rho_{a} \quad$ air density $\left(1.27 \mathrm{~kg} / \mathrm{m}^{3}\right)$

$\rho_{p} \quad$ produce density $\left(\mathrm{kg} / \mathrm{m}^{3}\right)$

$\tau \quad$ switching time $(s)$

$\tau_{f} \quad$ length of switching interval $(s)$

$A_{f} \quad$ floor area of the bulk $\left(m^{2}\right)$

$A_{p} \quad$ produce surface per bulk volume $\left(\mathrm{m}^{2} / \mathrm{m}^{3}\right)$

Bi Biot number $\frac{2 h R}{\lambda_{a}}$ (dimensionless)

$E \quad$ energy $(W)$

$L \quad$ bulk height $(m)$

$L_{2} \quad R \gamma(1-\gamma)$, char. length $(m)$

$M_{1} \quad \frac{\lambda_{p}}{\rho_{p} c_{p}}\left(m^{2} / s\right)$

$M_{2} \quad \frac{a}{c_{p}}(1 / s)$

$M_{3} \quad \sqrt{M_{2} / M_{1}} R$ (dimensionless)

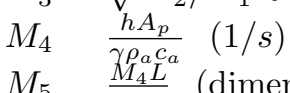

$M_{5} \quad \frac{M_{4} L}{v}$ (dimensionless)

$M_{6} \quad(1-\alpha) \exp \left(-M_{5}\left(B_{p}+A_{p}\right) / A_{p}\right) \quad$ (dimensionless)

$\mathrm{Nu} \quad$ Nusselt number $\frac{2 h R}{\lambda_{\alpha}}$ (dimensionless)

Pr Prandtl number $\frac{\nu}{\alpha_{t h}} \quad$ (dimensionless)

$R \quad$ product radius $(m)$

Re Reynolds number $\frac{v L_{2}}{\nu}$, see Xu and Burfoot [1999b]

$T_{a} \quad$ air temperature in the bulk $(K)$

$T_{c} \quad$ cooling element temperature $(K)$

$T_{\text {ini }}$ initial temperature $(K)$

$T_{p} \quad$ produce temperature

$V \quad$ volume of shaft $\left(\mathrm{m}^{3}\right)$

a product heat production $(W / k g K)$

$a_{1} \quad \mathrm{Bi}$ (dimensionless)

$a_{2} \quad 2 M_{3} \cot \left(M_{3}\right)-2+\mathrm{Bi} \quad$ (dimensionless)

$a_{3} \quad \frac{R^{2}}{M_{1}} \cot ^{2}\left(M_{3}\right)+\frac{R^{2}}{M_{1}}-\frac{M_{3}}{M_{2}} \cot \left(M_{3}\right)(s)$

$c_{a}$ heat capacity of air $\left(110^{3} \mathrm{~J} / \mathrm{kg} \mathrm{K}\right)$

$c_{p} \quad$ heat capacity of produce $(\mathrm{J} / \mathrm{kg} K)$

$h$ heat transfer coefficient $\left(W / m^{2} K\right)$

$v \quad$ air velocity inside the bulk $(\mathrm{m} / \mathrm{s})$ 